\title{
Fauna parasitária de Leporinus friderici (characidae) oriundo do Rio Mazagão, Amazônia Oriental
}

Nas regiões neotropicais, há estimativa de 9.100 espécies de peixes, e centenas de espécies novas são descritas anualmente, o qual a bacia amazônica abriga cerca de 2200 espécies de peixes corresponde a essa estimativa. Leporinus friderici é um peixe da ordem Characiformes conhecido popularmente como 'piau três pintas' ou 'aracú', trata-se de uma espécie abundante nas regiões neotropicais, que possui hábitos alimentares onívoros, se alimentando de material vegetal, detritos, frutos, sementes, camarão e pequenos peixes. É um peixe muito utilizado na alimentação pela qualidade de sua carne e é facilmente encontrado na região do Rio Matapí-AP. Foram realizadas coletas trimestrais durante setembro de 2015 a maio de 2016 e de agosto de 2017 a outubro de 2018 , onde foram capturados 49 espécimes de L. friderici. A fauna de L. friderici foi bastante diversificada, sendo composta por macro e microparasitos, infectando brânquias, intestino, fígado, vesícula biliar, coração, nadadeira e baço do hospedeiro. Durante as análises foram encontradas quatro taxas, sendo eles: Nematoda, Platyhelminthes, Cnidaria (Myxozoa) e Ciliophora, e nove grupos de parasitos, Anisakidae gen. sp., Contracaecum sp., Dactylogyridae gen. sp., larvas de digenea (metacercária), Henneguya sp., Myxobolus sp., Ceratomyxa sp., Piscinoodinium pillulare, Ichthyophthirius multifiliis, Trichodina sp. e Chilodonella sp.. A ocorrência de micropasitos eucariotos foi a primeira registrada para L. friderici do estado do Amapá.

Palavras-chave: Peixe; Parasitos; Amapá; Água doce; Prevalência.

\section{Parasitic fauna of Leporinus friderici (characidae) from the Mazagão River, East Amazon}

In the Neotropical regions, there are an estimated 9,100 species of fish, and all new species are annual, which the Amazon basin is home to about 2,200 species of fish corresponding to this estimate. Leporinus friderici is a fish of the order Characiformes popularly known as 'piau três pintas' or 'aracú', it is an abundant species in the Neotropical regions, which has omnivorous eating habits, feeding on plant material, debris, fruits, seeds, shrimp and small fish. It is a fish widely used in food for the quality of its meat and is easily found in the Rio Matapi-AP region. Quarterly collections were carried out during September 2015 to May 2016 and from August 2017 to October 2018, where 49 specimens of L. friderici were captured. The fauna of L. friderici was quite diverse, being composed of macro and microparasites, infecting gills, intestine, liver, gallbladder, heart, fin and spleen of the host. During the analysis, four taxa were found, namely: Nematoda, Platyhelminthes, Cnidaria (Myxozoa) and Ciliophora, and nine groups of parasites, Anisakidae gen. sp., Contracaecum sp., Dactylogyridae gen. sp., digenea larvae (metacercaria), Henneguya sp., Myxobolus sp., Ceratomyxa sp., Piscinoodinium pillulare, Ichthyophthirius multifiliis, Trichodina sp. and Chilodonella sp.. The occurrence of eukaryotic micropasites was the first recorded for L. friderici from the state of Amapá.

Keywords: Fish; Parasites; Amapá; Freshwater; Prevalence.

Topic: Organização da Biodiversidade

Reviewed anonymously in the process of blind peer.
Received: 02/02/2021

Approved: 27/02/2021
Abthyllane Amaral de Carvalho (iD)

Universidade Federal do Pará, Brasil

http://lattes.cnpq.br/1247132992493228

http://orcid.org/0000-0001-9598-1590

abthyllaneamaral@gmail.com

Raul Carvalho Júnior (iD)

Universidade Federal do Pará, Brasil

http://lattes.cnpq.br/2561967151223231

http://orcid.org/0000-0002-4433-6580

rauljrmacapa@gmail.com

Roger Leomar da Silva Ferreira (ic

Universidade Federal do Amapá, Brasil

http://lattes.cnpq.br/3436682345893190

http://orcid.org/0000-0001-5316-0380

rogersilfer@yahoo.com.br

\author{
Priscila Gomes de Araújo (D) \\ Universidade Federal do Amapá, Brasil \\ http://lattes.cnpq.br/6824184598311411 \\ http://orcid.org/0000-0001-8833-2170 \\ prizsla@hotmail.com \\ Edilson Rodrigues Matos (iD) \\ Universidade Federal do Amapá, Brasil \\ http://lattes.cnpq.br/7895814591867510 \\ http://orcid.org/0000-0002-9918-1408 \\ edilson.matos9@gmail.com \\ Marcela Nunes Videira (ii) \\ Universidade Federal do Amapá, Brasil \\ http://lattes.cnpq.br/0155583853689152 \\ http://orcid.org/0000-0002-9559-8521 \\ videiravet@gmail.com
}

\section{Referencing this:}

CARVALHO, A. A.; CARVALHO JÚNIOR, R.; FERREIRA, R. L. S.; ARAÚJO, P. G.; MATOS, E. R.; VIDEIRA, M. N.. Fauna parasitária de Leporinus friderici (characidae) oriundo do Rio Mazagão, Amazônia Oriental. Revista Ibero Americana de Ciências Ambientais, v.12, n.2, p.158166, 2021. DOI: http://doi.org/10.6008/CBPC21796858.2021 .002 .0016 


\section{INTRODUÇÃO}

O Brasil por possuir uma imensa zona costeira e diversos rios têm na pesca um importante papel econômico e social (ARAÚJO et al., 2014), tendo como estimativa de captura de 225 mil toneladas de peixes para pesca de interiores (FAO, 2018). Com o aumento na atividade pesqueira no Brasil, houve também a necessidade de se promover pesquisas que fomentem as discussões e auxiliem no conhecimento acerca desses recursos no país (OLIVEIRA et al., 2016).

De acordo com Reis et al. (2016), ainda não se conhece com exatidão o número de peixes que ocorrem nas regiões neotropicais, mas há estimativas de 9.100 espécies, e centenas de espécies novas são descritas anualmente, havendo um consenso que se trata da maior diversidade de peixes de água doce do mundo. A bacia amazônica abriga cerca de 2200 espécies de peixes (OBERDORFF et al., 2019). Leporinus friderici Bloch (1794), conhecido popularmente como piau-três-pintas/aracu piau, possui uma ampla distribuição na América do Sul ocorrendo no Suriname, Bacia Amazônica, rios do Nordeste, bacias do Rio Paraná, Paraguai e da Prata (SANCHES et al., 2001).

Este peixe, possui hábito alimentar onívoro, de comportamento generalista e oportunista (DURÕES et al., 2001), alimentando-se de insetos, detritos, frutos, sementes, camarão e pequenos peixes, bem como hábitos diurnos e sedentários (OLIVATTI et al., 2011). No sistema do Rio Amazonas, esse peixe se reproduz no período de abril a outubro, e tem desova total (OLIVEIRA et al., 2016) e está entre as mais produzidas no Brasil (IBGE, 2018), devido à alta qualidade da carne e fácil aceitação do mercado consumidor e pesca esportiva (SOUZA et al., 2020).

Essa espécie de peixe, assim como outras, sofre a ação de parasitos que, algumas vezes, podem causar sérios danos ao seu hospedeiro, e em determinadas situações podem ter potencial zoonótico, infectando humanos (SCHALCH et al., 2005; PAVANELLI et al., 2008). Esses vertebrados têm uma maior quantidade e variedade de parasitos do que qualquer outra classe, o que ocorre devido ao longo período que vivem em associação com uma variedade de formas de invertebrados (THATCHER, 1991).

Diversos fatores predispõem os peixes ao parasitismo, como sazonalidade, sexo, idade, tamanho do corpo, padrões de comportamento, hábito alimentar e disponibilidade de hospedeiros intermediários na dieta dos peixes (NEVES et al., 2013; TAVARES-DIAS et al., 2014). O parasitismo pode causar mudanças na eficiência de forrageamento, seleção de habitat, capacidade competitiva, relações predador-presas, desempenho de natação, escolha de companheiro e comportamento sexual (GOMIERO et al., 2012).

O objetivo desse trabalho foi realizar um levantamento da fauna parasitaria de Leporinus friderici da região do Rio Matapí em Macapá-AP, assim como determinar a prevalência e os sítios de infecção parasitária nos peixes.

\section{MATERIAIS E MÉTODOS}

\section{Área de estudo e coleta}

Rio Mazagão, localizado no município de Mazagão-AP, possui em suas margens vegetação do tipo 
floresta de várzea e floresta de igapó, que são ambientes com papel relevante para populações de peixes, servindo como fonte de alimento e abrigo (ZACARDI, 2016). A bacia hidrográfica desse rio, abrange área de $2.538 \mathrm{~km}^{2}$, passando por diversos municípios do estado do Amapá (TAKIYAMA et al., 2007).

Os espécimes de $L$. friderici foram coletados neste rio (Ponto 1: $0^{\circ} 08^{\prime} 22.2^{\prime \prime} \mathrm{N} 51^{\circ} 11^{\prime} 17.3^{\prime \prime} \mathrm{W}$; Ponto 2:

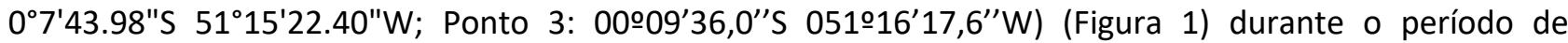
setembro de 2015 a maio de 2016 e em agosto de 2017 a outubro de 2018, em intervalos de três meses. Os peixes foram capturados com auxílio de rede de emalhar (30 mm entre nós opostos) pela equipe do grupo de pesquisa de Sanidade de Organismos Aquáticos da Amazônia da Universidade do Estado do Amapá (SOAAUEAP) e por pescadores locais.

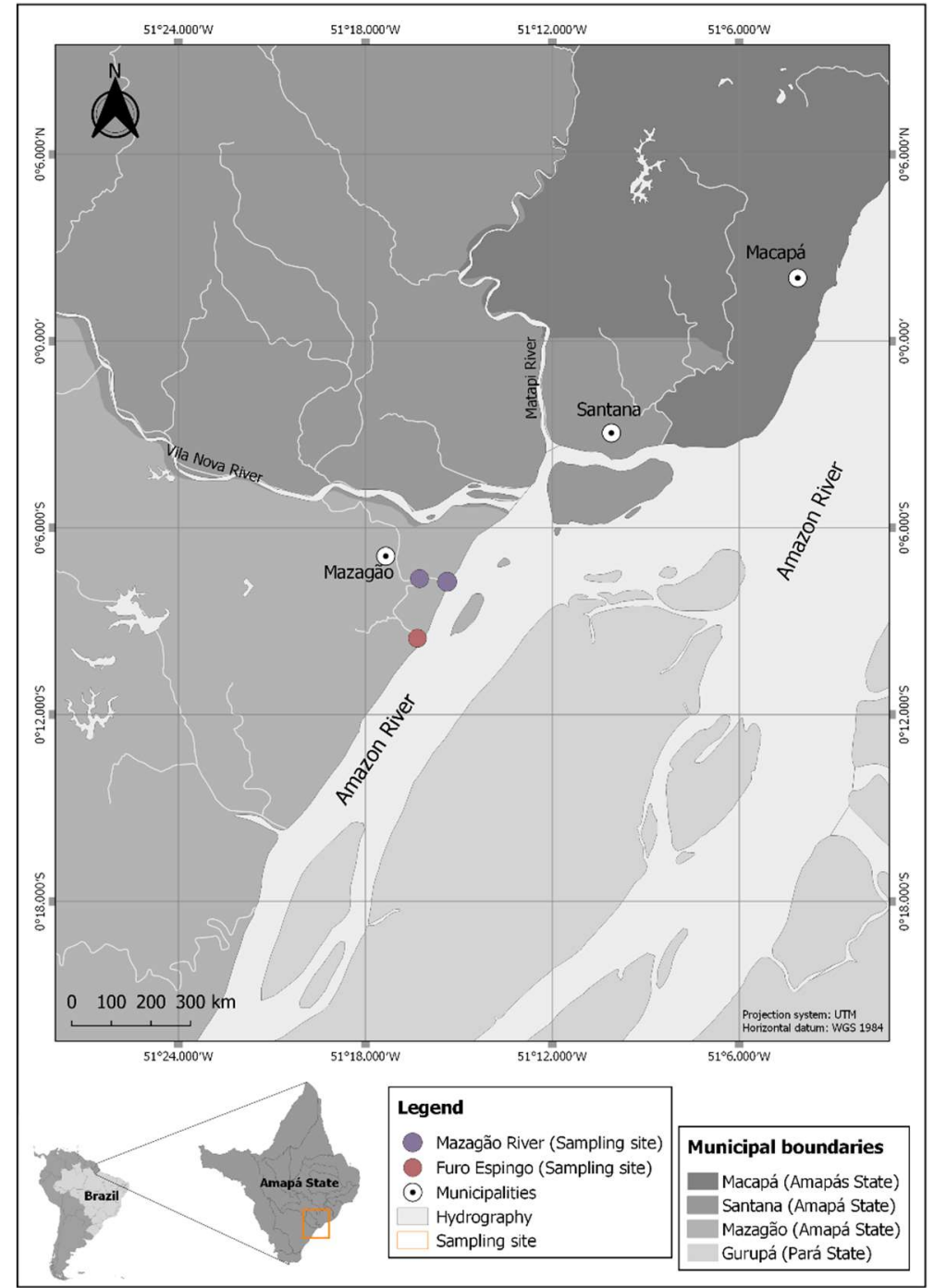

Figura 1: Mapa dos pontos de coletas de Leporinus friderici no município Mazagão, Amapá, Amazônia Oriental, Brasil.

Os exemplares foram transportados vivos em cubas térmicas com auxílio de aeração artificial até o Laboratório de Morfofisiologia e Sanidade Animal (LABMORSA) da UEAP, onde permaneceram até as análises parasitológicas. A pesquisa foi devidamente autorizada pelo Comitê de Ética de Uso Animal (CEUA) da Empresa Brasileira de Pesquisa Agropecuária (EMBRAPA-AP) sob o no 012/2018 e registrado no Sistema de Autorização e Informação da Biodiversidade (SISBIO/ICMBIO), licença no 50376-1. 


\section{Procedimentos e amostragem de peixes}

Os peixes foram dessensibilizados por meio de secção medular, com auxílio de instrumento metálico pontiagudo. Antes de iniciar as análises parasitológicas, peso $(\mathrm{g})$ e comprimento total $(\mathrm{cm})$ foram aferidos. Em microscópio estereoscópico binocular, toda a superfície corporal foi analisada, a fim de verificar a existência de lesões/cistos ou perda de revestimento. Em seguida, foi realizada uma incisão na região ventral, do orifício anal até o opérculo, com intuito de analisar cada órgão interno.

A metodologia para coleta e fixação de parasitos seguiu as recomendações de Eiras et al. (2006) e o índice parasitário de prevalência (\%) foi calculado com o proposto do Bush et al. (1997).

\section{RESULTADOS E DISCUSSÃO}

Foram coletados 49 espécimes de $L$. friderici com comprimento médio total de $17,20 \pm 2,6 \mathrm{~cm}$ e peso médio de 75,37 $\pm 33,2$ g. Durante as análises foram encontrados quatro taxas, sendo eles: Nematoda, Platyhelminthes, Cnidaria (Myxozoa) e Ciliophora. E nove grupos de parasitos, Anisakidae gen. sp. Skrjabin et al. (1945), Contracaecum sp. Railliet et al. (1912), Dactylogyridae gen. sp. Bychowsky (1933), larvas de digenea (metacercária), Henneguya sp. Thélohan (1892), Myxobolus sp. Bütschli (1882), Ceratomyxa sp. Thélohan (1892), Piscinoodinium pillulare Schäperclaus (1954), Ichthyophthirius multifiliis Fouquet (1876), Trichodina sp. Ehrenberg (1838) e Chilodonella sp. Strand (1928) (Tabela 1).

Tabela 1: Parasitos de Leporinus friderici do rio Matapi, Amazônia Oriental. SI: Sitio de Infecção; P: Prevalência.

\begin{tabular}{|c|c|c|}
\hline Parasito & SI & $\mathrm{P}(\%)$ \\
\hline Anisakidae gen. sp. & Fígado & 5,13 \\
\hline Contracaecum sp. & Intestino & 3,84 \\
\hline Larva de digenea (Metacercaria) & Fígado, Vesícula Biliar, Coração, Intestino, Brânquias, Nadadeira & 58,97 \\
\hline Dactylogyridae gen. sp. & \multirow{5}{*}{ ( } & 74,36 \\
\hline Piscinoodinium pilullare & & 30,77 \\
\hline Chillodonela sp. & & 2,56 \\
\hline Trichodina sp. & & 2,56 \\
\hline Ichthyophthirius multifiliis & & 5,13 \\
\hline Henneguya sp. & Brânquias, Coração, Vesícula biliar, Baço, Fígado & 58,97 \\
\hline Myxobolus sp. & Brânquias, Fígado, Vesícula biliar & 41,03 \\
\hline Ceratomyxa sp. & Vesícula biliar & 12,82 \\
\hline
\end{tabular}

A fauna endohelmíntica foi composta por platelmintos (Monogenea e Digenea) e larvas de nematoides, também observados em estudo de Travassos et al. (1928), Guidelli et al. (2006), Takemoto et al. (2009) e Yamada et al. (2017) na mesma espécie de hospedeiro.

Larvas de Contracaecum sp. apresentaram prevalência de $70 \%$ e também foram encontradas em $L$. friderici do Rio Paraná, descrito por Guidelli et al. (2006) com prevalência de 3,84\%. Outras larvas de nematoides da família Anisakidae também foram encontradas no fígado do hospedeiro com prevalência de 5,13\%. O gênero de Contracaecum é gênero mais prevalente em peixes de água doce da família Anisakidae. Essa família possui 24 gêneros, e alguns gêneros têm sido alvos de estudos, devido serem espécies responsáveis por causar zoonoses, conhecidas como anisaquíases (MARTINS et al., 2004; UBEIRA et al., 2000).

Dactylogyridae gen. sp. foram os parasitos que apresentaram a maior prevalência $(74,36 \%)$ entre os 
encontrados neste estudo, tendo como órgão infectado as brânquias. Três espécies da família Dactylogyridae foram encontradas nas brânquias de Leporinus macrocephalus no estado do Acre nas análises de Martins (2017). Já no estudo de Zago et al. (2017), a prevalência em L. friderici por Tereancistrum flabellum Zago et al., 2017 foi de 15,5\%, e prevalência de 8,65\% em L. friderici estudado por Guidelli et al. (2006). Monogenoides da família Dactylogyridae são parasitos capazes de causar inúmeros prejuízos à criação de peixes por possuir hábitos alimentares que comprometem os tecidos, disseminando agentes causadores de doenças nos peixes em ambientes (PAVANELLI et al., 2008).

As larvas de digenéticos (metacercárias) foram encontradas em diversos órgãos do peixe, por apresentar baixa especificidade e rápida adaptação ao ambiente (TAKEMOTO et al., 2009). Esse parasito já foi descrito L. friderici de várias regiões do Brasil (TRAVASSOS et al., 1928; GUIDELLI et al., 2006; TAKEMOTO et al., 2009; YAMADA et al., 2017), inclusive em rios do estado do Amapá (OLIVEIRA et al., 2016).

Microparasitos eucariotos do subfilo Myxozoa foram encontrados neste estudo, tais como Henneguya sp. (58,97\%), Myxobolus sp. (41,03\%) e Ceratomyxa sp. (12,82\%), infectando diversos órgãos de L. friderici.

Para espécies de Leporinus spp. não há relatos na literatura de parasitismo por Myxobolus sp., porém já foi descrito para diversas espécies de peixes amazônicos, como em Pimelodus ornatus Kner (1858) por Myxobolus sp. (13,9\%) (MATOS et al., 2001), em Colossoma macropomum Cuvier (1816) por Myxobolus sp. (5,5\%) (MACIEL et al., 2011), em Eigenmannia sp por Myxobolus sp. (70\%) (SINDEAUX NETO et al., 2016), em Astyanax aff. bimaculatus por Myxobolus sp. (20\%) (SILVA et al., 2019) e em Thoracocharax stellatus Kner, 1858 por Myxobolus sp. (66,6\%) (ARAÚJO et al., 2018).

Em L. friderici já foi registrado a infecção por Henneguya friderici Casal et al. (2003) em L. friderici do rio Amazonas com prevalência de 30\% (CASAL et al., 2003) e em L. friderici do rio Mogi Guaçú-SP infectando 23,8\% das brânquias (VIDAL et al., 2017). Outras espécies de Henneguya spp. foram descritas para o gênero Leporinus , como Henneguya visibilis Moreira et al. (2014) (62,5\%) em Leporinus obtusidens Valenciennes, 1837 do rio Mogi Guaçu-SP (MOREIRA et al., 2014), Henneguya leporinicola Martins et al. (1999) em Leporinus macrocephalus Garavello et al. (1988) (7,14\%) cultivado em Santa Crus da Conceição-SP (CAPODIFOGLIO et al., 2015) e em L. macrocephalus cultivado em Capivari-SP (MARTINS et al., 1999), Henneguya azevedoi Barassa et al. (2012) (70\%) em L. obtusidens de rio Mogi Guaçu-SP (BARASSA et al., 2012), Henneguya caudicula Eiras et al. (2008) (33,3\%) em Leporinus lacustris Amaral Campos (1945) do rio Paraná (EIRAS et al., 2008) e Henneguya sp. em L. obtusidens (100\%) e L. friderici (100\%) do rio Paraná (PAVANELLI et al., 1998).

Até o presente momento, não houve trabalhos em Leporinus spp. que relatem a presença de parasitos do gênero Ceratomyxa, sendo essa a primeira ocorrência desse gênero. Porém espécies de Ceratomyxa spp. já foram reportadas em peixes amazônicos, como em Hemiodus microlepis Kner (1858) por Ceratomyxa microlepis (36\%) (AZEVEDO et al., 2013), em Hemiodus unimaculatus Bloch (1794) por Ceratomyxa fonsecai (16\%) (SILVA et al., 2020), em Brachyplatystoma rousseauxii Castelnau (1855) por Ceratomyxa gracílima (70\%) (ZATTI et al., 2018), em Cichla monoculus Agassiz (1831) por Ceratomyxa 
brasiliensis (3\%) (ZATTI et al. 2017), em Symphysodon discus Heckel (1840) por Ceratomyxa amazonensis (73,3\%) (MATHEWS et al., 2016) e em C. macropomum por Ceratomyxa vermiformis (ADRIANO et al., 2016).

A fauna parasitária de L. friderici, nesse estudo, também foi composta por protozoários ciliados parasitando as brânquias, como o Piscinoodinium pillulare com prevalência de 30,77\%, Ichthyophthirius multifiliis com 5,13\%, Chilodonella sp. em 2,56\% e Trichodina sp. em 2,56\% dos peixes. Protozoários parasitas em peixes são observados causando graves infecções nos hospedeiros e perdas no cultivo (PAVANELLI et al., 2008). P. pillulare, I. multifiliis e Trichodina sp. já foram relatados em L. friderici da bacia do Igarapé da Fortaleza em Macapá-AP.

P. pillulare, I. multifiliis e Trichodina sp. já foram encontrados em L. macrocephalus cultivados em Franca-SP (TAVARES-DIAS et al., 1999), em Guariba-SP (SCHALCH et al., 2006) e em outros municípios do estado de São Paulo-Br (MARTINS et al., 2001).

O protozoário Chilodonella sp. é bastante encontrado em peixes do continente asiático (URAWA et al., 1992; OKAMURA et al., 2014; Ll et al., 2018; ZHANG et al., 2018; WANG et al., 2019), porém já foi relatado em alguns peixes no Brasil como nas brânquias de Xiphophorus maculatus Günther, 1866 (PIAZZA et al., 2006) e nas brânquias do hibrido "patinga" (P. mesopotamicus x P. brachypomus) (FRANCESCHINI et al., 2013), mas sem registros, até o presente estudo, em L. friderici.

\section{CONCLUSÕES}

A fauna parasitária de L. friderici foi bastante diversificada, sendo composta por macro e microparasitos, infectando vários órgãos no hospedeiro. Neste estudo, foi observado pela primeira vez, a presença de microparasitos eucariotos do gênero Ceratomyxa sp. e Myxobolus sp., assim com o protozoário, Chilodonella sp., e os demais parasitos como mais um relato para esta espécie de peixe, desta vez no estado do Amapá.

\section{REFERÊNCIAS}

ADRIANO, E. A.; OKAMURA, B.. Motility, morphology and phylogeny of the plasmodial worm, Ceratomyxa vermiformis n.sp.(Cnidaria: Myxozoa: Myxosporea). Parasitology, v.144, n.2, p.158-168, 2016. DOI: https://doi.org/10.1017/S0031182016001852

ARAÚJO, M. V. L. F.; SILVA, K. C. A.; SILVA, B. B.; FERREIRA, I. L. S.; CINTRA, I. H. A.. Pesca e procedimentos de captura do Camarão-da- Amazônia a jusante de uma Usina Hidrelétrica na Amazônia Brasileira. Biota Amazônia, v.4, n.2, p.102-112, 2014. DOI: http://dx.doi.org/10.18561/2179-

5746/biotaamazonia.v4n2p102-112

ARAÚJO, R. S.; CORRÊA, F.; SOUSA, F. B.; RAMOS, A. B. M. A. NETO, J. L. S.; MATOS, E. R.. Ocorrência de Myxobolus sp. (Myxozoa) em Thoracocharax stellatus (Kner, 1858) (Characiformes) em um igarapé da floresta amazônica, Pará, Brasil. Brazilian Journal of Aquatic Science and Technology, v.21, n.1, p.16-20, 2018.

AZEVEDO, C.; ROCHA, S.; CASAL, G.; CLEMENTE, S. C. S.; MATOS, P.; AL-QURAISHY, S.; MATOS, E.. Ultrastructural description of Ceratomyxa microlepis sp.nov. (Phylum Myxozoa): a parasite infecting the gall bladder of Hemiodus microlepis, a freshwater teleost from the Amazon River. Memórias do Instituto Oswaldo Cruz, v.108, n.2, p.150-154, 2013. DOI: http://dx.doi.org/10.1590/0074$\underline{0276108022013004}$

BARASSA, B.; ADRIANO, E. A.; CORDEIRO, N. S.; ARANA, S.; CECCARELLI, P. S.. Morphology and host-parasite interaction of Henneguya azevedoi n. sp., parasite of gills of Leporinus obtusidens from Mogi-Guaçu River, Brazil. Parasitology Research, v.110, n.2, p.887-894, 2012. DOI: http://doi.org/10.1007/s00436-011-2571-5

BUSH, A. O.; LAFFERTY, K. D.; LOTZ, J. M.; SHOSTAK, A. W.. Parasitology meets ecology on its own terms: Margolis et al. revisited. The Journal ofP, v.83, n.4, p.575-583, 1997. DOI: http://doi.org/10.2307/3284227

CAPODIFOGLIO, K. R. H.; ADRIANO, E. A.; SILVA, M. R. M.; MAIA, A. A. M.. Supplementary data of Henneguya leporinicola (Myxozoa, Myxosporea) a parasite of Leporinus 
macrocephalus from fish farms in the state of São Paulo, Brazil. Acta Parasitologica, v.60, n.3, p.451-458, 2015. DOI: https://doi.org/10.1515/ap-2015-0062

CASAL, G.; MATOS, E.; AZEVEDO, C.. Light and electron microscopic study of the myxosporean, Henneguya friderici sp.from the Amazonian teleostean fish, Leporinus friderici. Parasitology, v.126, n.4, p.313-319. 2003. DOI: https://doi.org/10.1017/S0031182003002944

DURÕES, R.; POMPEU, P. S.; GODINHO, A. L.. Alimentação de quatro espécies de Leporinus (Characiformes, Anostomidae) durante a formação de um reservatório no sudeste do Brasil. Iheringia. Série Zoologia, n.90, p.183-191, 2001. DOI: https://doi.org/10.1590/S0073-47212001000100019

EIRAS, J. C.; TAKEMOTO, R. M.; PAVANELLI, G. C.. Métodos de estudo e técnicas laboratoriais em parasitologia de peixes. Maringá: Eduem, 2006.

EIRAS, J. C.; TAKEMOTO, R. M.; PAVANELLI, G. C.. Henneguya caudicula n.sp.(Myxozoa, Myxobolidae) a Parasite of Leporinus lacustris (Osteichthyes, Anostomidae) From the High Paraná River, Brazil, With a Revision of Henneguya spp.Infecting South American Fish. Acta Protozoologica, v.47, p.149-154, 2008.

FAO. Food and Agriculture Organization. The State of World Fisheries and Aquaculture 2018: Meeting the sustainable development goals. Romeu: FAO, 2018.

FRANCESCHINI, L.; ZAGO, A. C.; SCHALCH, S. H. C.; GARCIA, F.; ROMERA, D. M.; SILVA, R. J. D.. Parasitic infections of Piaractus mesopotamicus and hybrid (P. mesopotamicus $\mathrm{X}$ Piaractus brachypomus) cultured in Brazil. Revista Brasileira de Parasitologia Veterinária, v.22, n.3, p.407-414, 2013. DOI: http://doi.org/10.1590/S1984-29612013000300015

GOMIERO, L. M. I.; SOUZA, U. P.; BRAGA, F. M. S. I.. Condition factor of Astyanax intermedius Eigenmann, 1908 (OSTEICHTHYES, CHARACIDAE) parasitised by Paracymothoa astyanax Lemos de Castro, 1955 (CRUSTACEA, CYMOTHOIDAE) in the Grande River, Serra do Mar State Park - Santa Virgínia Unit, São Paulo, Brazil. Brasiliana Jornal of Biology, São Carlos, v.72, n.2, p.379-388, 2012. DOI: http://dx.doi.org/10.1590/S1519-69842012000200020

GUIDELLI, G.; TAVECHIO, W. L. G.; TAKEMOTO, R. M.; PAVANELLI, G. C.. Fauna parasitária de Leporinus lacustris e Leporinus friderici (Characiformes, Anostomidae) da planície de inundação do alto rio Paraná, Brasil. Acta Scientiarum. Biological Sciences, v.28, n.3, p.281-290, 2006.

IBGE. Instituto brasileiro de geografia e estatística. Produção da pecuária municipal de 2017. Rio de Janeiro: IBGE, 2018.

LI, M.; WANG, R.; GOMES, G. B.; ZOU, H.; LI, W. X.; WU, S. G.; WANG, G. T.; PONCE-GORDO, F.. Epidemiology and identification of two species of Chilodonella affecting farmed fishes in China. Veterinary parasitology, v.264, P.8-17, 2018. DOI: https://doi.org/10.1016/j.vetpar.2018.10.009

MACIEL, P. O.; AFFONSO, E. G.; BOIJINK, C. D. L.; TAVARESDIAS, M.; INOUE, L. A. K. A.. Myxobolus sp. (Myxozoa) in the circulating blood of Colossoma macropomum (Osteichthyes, Characidae). Revista Brasileira de Parasitologia Veterinária, v.20, n.1, p.82-84, 2011. DOI:

https://doi.org/10.1590/S1984-29612011000100018

MARTINS, M. L.; SOUZA, V. N.; MORAES, J. R.; MORAES, F. R.. Gill infection of Leporinus macrocephalus Garavello \& Britski, 1988 (Osteichthyes: Anostomidae) by Henneguya leporinicola n.sp.(Myxozoa: Myxobolidae). Description, histopathology and treatment. Revista Brasileira de Biologia, v.59, n.3, p.527-534, 1999. DOI: https://doi.org/10.1590/S0034-71081999000300018

MARTINS, M. L.; MORAES, J. R. E.; ANDRADE, P. M.; SCHALCH, S. H. C.; MORAES, F. R. D.. Piscinoodinium pillulare (Schäperclaus, 1954) Lom, 1981 (Dinoflagellida) infection in cultivated freshwater fish from the Northeast region of São Paulo State, Brazil: parasitological and pathological aspects. Brazilian Journal of Biology, v.61, n.4, p.639-644, 2001. DOI: https://doi.org/10.1590/S1519-69842001000400013

MARTINS, M. L.; TAVARES-DIAS, M.; FUJIMOTO, R. Y.; ONAKA, E. M.; NOMURA, D. T.. Haematological alterations of Leporinus macrocephalus (Osteichtyes: Anostomidae) naturally infected by Goezia leporini (Nematoda: Anisakidae) in fish pond. Arquivo Brasileiro de Medicina Veterinária e Zootecnia, v.56, n.5, p.640-646, 2004. DOI: https://doi.org/10.1590/S0102-09352004000500011

MATHEWS, P. D.; NALDONI, J.; MAIA, A. A.; ADRIANO, E. A.. Morphology and small subunit rDNA-based phylogeny of Ceratomyxa amazonensis n.sp.parasite of Symphysodon discus, an ornamental freshwater fish from Amazon.Parasitology Research, v.115, n.10, p.4021-4025, 2016. DOI: https://doi.org/10.1007/s00436-016-5173-4

MATOS, E. R.; CORRAL, L.; MATOS, P.; CASAL, G.; AZEVEDO, C.. Incidência de parasitas do Philum Myxozoa (Sub-reino Protozoa) em peixes da região amazônica, com especial destaque para o gênero Henneguya. Revista Ciências Agrarias, v.36, p.83-99, 2001.

MOREIRA, G. S.; ADRIANO, E. A.; SILVA, M. R.; CECCARELLI, P. S.; MAIA, A. A.. Morphology and $18 \mathrm{~S}$ rDNA sequencing identifies Henneguya visibilis n.sp., a parasite of Leporinus obtusidens from Mogi Guaçu River, Brazil. Parasitology Research, v.113, n.1, 81-90, 2014. DOI: http://doi.org/10.1007/s00436-013-3629-3

NEVES, L. R.; PEREIRA, F. B.; TAVARES-DIAS, M.; LUQUE J. L. Seasonal Influence on the Parasite Fauna of a Wild Population of Astronotus ocellatus (Perciformes: Cichlidae) from the Brazilian Amazon.The Journal of Parasitology, v.99, n.4, p.718-721, 2013. DOI: https://doi.org/10.1645/12-84.1

OBERDORFF, T.; DIAS, M. S.; JÉZÉQUEL, C.; ALBERT, J. S.; ARANTES, C. C.; BIGORNE, R.; FERNANDO M.; VALLEROS, C.; WEVER, A.; FREDERICO, R. G.; HIDALGO, M.; HUGUENY, B.; LEPRIEUR, F.; MALDONADO, M.; OCAMPO, J. M.; MARTENS, K.; ORTEGA, H.; SARMIENTO, J.; TEDESCO, P. A.; VILARA, G. T.; WINEMILLER, K. O.; ZUANON, J.. Unexpected fish diversity gradients in the Amazon basin. Science Advances, v.5, n.9, p.81-86, 2019. DOI:

http://doi.org/10.1126/sciadv.aav8681

OKAMURA, T.; KUWAMURA, K.; OGAWA, K.. Mortality of wild three-lips Opsariichthys uncirostris by infection with Chilodonella hexasticha. Gyobyo Kenkyu: Fish Pathology, 
v.49, n.2, p.49-52, 2014. DOI:

http://dx.doi.org/10.3147/isfp.49.49

OLIVATTI, A. M.; BONI, T. A.; SILVA JÚNIOR, N. J.; RESENDE, L. V.; GOUVEIA, F. O.; TELLES, M. P. C.. Heterologous amplification and characterization of microsatellite markers in the Neotropical fish Leporinus friderici. Genetics and Molecular Research, Ribeirão Preto, v.10, n.3, p.1403-1408, 2011.

OLIVEIRA, M. B.; GONÇALVES, R. A.; FERREIRA, D. O.; PINHEIRO, D. A.; NEVES, L. R.; DIAS, M. K.; TAVARES-DIAS, M.. Parasitos metazoários de duas populações de Leporinus friderici (Characiformes, anostomidae) do sistema do Rio Amazonas no Brasil. In: ENCONTRO BRASILEIRO DE PATOLOGISTAS DE ORGANISMOS AQUÁTICOS, 14. Anais. Florianópolis: UFSC, 2016.

PAVANELLI, G. C.; EIRAS, J. C.; SARAIVA, A.. Henneguya spp. (Myxozoa, Myxosporea, Myxobolidae) parasitizing fishes from Paraná river, Brazil. Acta Scientiarum. Biological Sciences, v.20, p.161-163, 1998. DOI: https://doi.org/10.4025/actascibiolsci.v20i0.4467

PAVANELLI, G. C.; EIRAS, J. C.; TAKEMOTO, R. M.. Doenças de peixes: profilaxia, diagnóstico e tratamento. 3 ed. Maringá: EDUEM, 2008.

PIAZZA, R. S.; MARTINS, M. L.; GUIRALDELLI, L.; YAMASHITA M. M.. Parasitic diseases of freshwater ornamental fishes commercialized in Florianópolis, Santa Catarina, Brazil. Boletim do Instituto de Pesca, v.32, n.1, p.51-57, 2006.

REIS, R. E.; ALBERT, J. S.; DI DARIO, F.; MINCARONE, M. M.; PETRY, P.; ROCHA, L. A.. Fish biodiversity and conservation in South America. Journal of Fishbiology, v.89, n.1, p.12-47, 2016. DOI: http://doi.org/10.1111/jfb.13016

SANCHES, P. V.; BAUMGARTNER, G.; BIALETZKI, A.; SUIBERTO, M. R.; GOMES, F. D. C.; NAKATANI, K.; BARBOSA, N. D. C.. Caracterização do desenvolvimento inicial de Leporinus friderici (Osteichthyes, Anostomidae) da bacia do rio Paraná, Brasil. Revista Acta Scientiarum, Maringá, v.23, n.2, p.383-389, 2001. DOI:

https://doi.org/10.4025/actascibiolsci.v23i0.2693

SCHALCH, S. H. C.; MORAES, F. R.. Distribuição sazonal de parasitos branquiais em diferentes espécies de peixes em pesque-pague do município de Guariba- SP, Brasil. Revista Brasileira Parasitologia Veterinaria, v.14, n.4, p.141-146, 2005.

SCHALCH, E. H.; MORAES, F. R.; MORAES, J. R.. Efeitos do parasitismo sobre a estrutura branquial de Leporinus macrocephalus Garavello e Britsk, 1988 (Anastomidae) e Piaractus mesopotamicus Holmberg, 1887 (Osteichthyes: Characidae). Revista Brasileira de Parasitologia Veterinária, v.15, n.3, p.110-115, 2006.

SILVA, M. F. D.; SOUSA-HENRIQUE, D. D. D.; MESSIAS-LUZ, N.; BORRALHO, L. D. S.; OLIVEIRA, J. D. D.; SINDEAUX NETO, J. L.; MATOS, E. R.. Myxobolus sp.(Myxozoa; Myxosporea) causing asymptomatic parasitic gill disease in Astyanax aff. bimaculatus (Characiformes; Characidae) in the Tocantins River Basin, Amazon Region, Brazil. Revista Brasileira de Parasitologia Veterinária, v.28, n.4, p.739-743, 2019. DOI: https://doi.org/10.1590/s1984-29612019041
SILVA, M. F.; CARVALHO, A. E. F. B.; HAMOY, I.; MATOS, E. R.. Coelozoic parasite of the family Ceratomyxidae (Myxozoa, Bivalvulida) described from motile vermiform plasmodia found in Hemiodus unimaculatus Bloch, 1794. Parasitology Research, v.119, p.871-878, 2020. DOI: https://doi.org/10.1007/s00436-019-06505-5

SINDEAUX NETO, J. L.; VELASCO, M.; SILVA, J. M. V. D.; SANTOS, P. D. F. S. D.; SANCHES, O.; MATOS, P.; MATOS, E.. Lymphocytic meningoencephalomyelitis associated with Myxobolus sp.(Bivalvulidae: Myxozoa) infection in the Amazonian fish Eigenmannia sp.(Sternopygidae: Gymnotiformes). Revista Brasileira de Parasitologia Veterinária, v.25, n.2, p.158-162, 2016. DOI: https://doi.org/10.1590/S1984-29612016023

SOUZA, T. G. D.; KURADOMI, R. Y.; RODRIGUES, S. M.; BATLOUNI, S. R.. Wild Leporinus friderici induced spawning with different dose of $\mathrm{mGnRHa}$ and metoclopramide or carp pituitary extract. Animal Reproduction, v.17, n.1, 2020. DOI: https://doi.org/10.21451/1984-3143-ar2019-0078

TAKEMOTO, R. M.; PAVANELLI, G. C.; LIZAMA, M. A. P.; LACERDA, A. C. F.; YAMADA, F. H.; CESCHINI, T. L.; BELLAY, S.. Diversity of parasites of fish the upper Paraná River floodplain, Brazil. Brazilian Journal of Biology, v.69, p.691705, 2009. DOI: https://doi.org/10.1590/S1519$\underline{69842009000300023}$

TAKIYAMA, L. R.; CUNHA, A. C.; SILVA, M. S.; MARTINS, M. H. A.; RODRIGUES, L.; JC, A.. Subsídios à gestão de recursos hídricos na bacia hidrográfica do Rio Matapi. Relatório Técnico (Processo CNPq no 504083/2003-1). Macapá: IEPA, 2007.

TAVARES-DIAS, M.; SCHALCH, S. H. C.; MARTINS, M. L.; SILVA, É. D.; MORAES, F. R.; PERECIN, D.. Hematologia de teleósteos brasileiros com infecção parasitária. I. Variáveis do Leporinus macrocephalus Garavelo e Britski, 1988 (Anostomidae) e Piaractus mesopotamicus Holmberg, 1887 (Characidae). Acta Scientiarum. Biological Sciences, v.21, p.337-342, 1999. DOI: https://doi.org/10.4025/actascibiolsci.v21i0.4440

TAVARES-DIAS, M.; OLIVEIRA, M. S. B.; GONÇALVES, R. A.; SILVA, L. M. A.. Ecology and seasonal variation of parasites in wild Aequidens tetramerus, a Cichlidae from the Amazon. Acta Parasitologica, v.54, n.1, p.158-164, 2014. DOI: https://doi.org/10.2478/s11686-014-0225-3

THATCHER, V. E.. Amazon fish parasites. Amazoniana, v.11, n.3-4, p.263-572, 1991

TRAVASSOS, L.; ARTIGAS, P. T.; PEREIRA, C.. Fauna helmintológica dos peixes de água doce do Brasil. Arquivos do Instituto Biológico, São Paulo, v.1, p.5-67, 1928.

UBEIRA, F. M.; IGLESIAS, R.. Monoclonal antibodies in the study of Anisakis simplex. Allergy, v.55, n.59, p.18-27, 2000. DOI: http://dx.doi.org/10.1034/j.1398-9995.2000.03404.x

URAWA, S.; YAMAO, S.. Scanning electron microscopy and pathogenicity of Chilodonella piscicola (Ciliophora) on juvenile salmonids. Journal of Aquatic Animal Health, v.4, n.3, p.188-197, 1992. DOI: https://doi.org/10.1577/15488667(1992)004\%3C0188:SEMAPO\%3E2.3.CO;2 
VIDAL, L. G. P.. Myxozoa Grassé, 1970 (Cnidaria:

Myxosporea): Sinopse das espécies parasitando peixes nas Américas e diagnóstico morfológico e molecular das espécies parasitando Characiformes, Leporinus friderici (Anostomidae) e Astyanax altiparanae (Characidae) oriundos do rio Mogi Guaçú, São Paulo, Brasil. Tese (Doutorado em Ciências Veterinárias) - Universidade Federal Rural do Rio de Janeiro, Seropédica, 2017.

WANG, Z.; ZHOU, T.; YANG, H.; GU, Z.. First diagnosis of ectoparasitic ciliates (Trichodina and Chilodonella) on farmed juvenile yellow catfish, Tachysurus fulvidraco in China. Aquaculture Research, v.50, n.11, p.3275-3285, 2019. DOI: https://doi.org/10.1111/are.14285

YAMADA, F. H.; BONGIOVANI, M. F.; YAMADA, P. O.; SILVA, R. J.. Parasite infra communities of Leporinus friderici: A comparison of three tributaries of the Jurumirim Reservoir in southeastern Brazil. Anais da Academia Brasileira de Ciências, v.89, n.2, p.953-963, 2017. DOI: https://doi.org/10.1590/0001-3765201720160554

ZACARDI, D. M.. Social aspects and activity fisheries technical use in Tracajatuba River, Amapá, Brazil. Acta of Fisheries and Aquatic Resources, v.3, n.2, p.31-48, 2016.
ZAGO, A. C.; YAMADA, F. H.; FRANCESCHINI, L.; BONGIOVANI, M. F.; YAMADA, P. O. F.; SILVA, R. J.. Uma nova espécie de Tereancistrum (Monogenea, Dactylogyridae) das brânquias de três espécies de Leporinus (Characiformes, Anostomidae) e uma descrição revisada de Tereancistrum parvus. Anais da Academia Brasileira de Ciências, v.89, n.2, p.1121-1131, 2017. DOI: http://dx.doi.org/10.1590/0001-3765201720160628

ZATTI, S. A.; ATKINSON, S. D.; BARTHOLOMEW, J. L.; MAIA, A. A.; ADRIANO, E. A.. Amazonian waters harbour an ancient freshwater Ceratomyxa lineage (Cnidaria: Myxosporea). Acta tropica, v.169, p.100-106, 2017. DOI: http://doi.org/10.1016/j.actatropica.2017.02.006

ZATTI, S. A.; ATKINSON, S. D.; MAIA, A. A.; BARTHOLOMEW, J. L.; ADRIANO, E. A.. Ceratomyxa gracillima n.sp.(Cnidaria: Myxosporea) provides evidence of panmixia and ceratomyxid radiation in the Amazon basin. Parasitology, v.145, n.9, p.1137-1146, 2017. DOI: https://doi.org/10.1017/\$0031182017002323

ZHANG, T.; WANG, C.; KATZ, L. A.; GAO, F.. A paradox: rapid evolution rates of germline-limited sequences are associated with conserved patterns of rearrangements in cryptic species of Chilodonella uncinata (Protista, Ciliophora). Science China Life Sciences, v.61, n.9, p.1071-1078, 2018.

A CBPC - Companhia Brasileira de Produção Científica (CNPJ: 11.221.422/0001-03) detém os direitos materiais desta publicação. Os direitos referem-se à publicação do trabalho em qualquer parte do mundo, incluindo os direitos às renovações, expansões e disseminações da contribuição, bem como outros direitos subsidiários. Todos os trabalhos publicados eletronicamente poderão posteriormente ser publicados em coletâneas impressas sob coordenação da Sustenere Publishing, da Companhia Brasileira de Produção Científica e seus parceiros autorizados. Os (as) autores (as) preservam os direitos autorais, mas não têm permissão para a publicação da contribuição em outro meio, impresso ou digital, em português ou em tradução. 